\title{
¿Hay discriminación en contra de las mujeres en el mercado laboral ecuatoriano?
}

\section{Alberto Posso}

International Trade and Development Group, Economics, EFM, Royal Melbourne Institute of Technology (RMIT), Melbourne, Australia

Recibido el 12 de mayo de 2015; aceptado el 28 de octubre de 2015

Disponible en Internet el 3 de diciembre de 2015

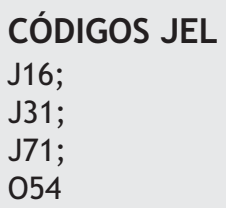

\section{PALABRAS CLAVE}

Desigualdad

de género;

Salarios;

Informalidad;

Subempleo;

Ecuador

\section{JEL CLASSIFICATION \\ J16; \\ $\mathrm{J} 31 ;$ \\ $\mathrm{J} 71$ \\ 054}

\section{KEYWORDS}

Gender inequality; Wages;

Informality;

Underemployment;

Ecuador

\begin{abstract}
Resumen La mayoría de los estudios sobre la desigualdad de género abordan exclusivamente las diferencias salariales. Este trabajo tiene como objetivo corregir este sesgo utilizando factores como el empleo, la formalidad de empleo y el subempleo. Ecuador es elegido como el escenario de este estudio, ya que goza de una estructura laboral relativamente única en América Latina. Utilizando datos de encuesta a nivel de hogar, este estudio encuentra evidencia de discriminación en el mercado laboral en contra de las mujeres en la forma de salarios más bajos y disparidades en materia de empleo, subempleo y formalidad. Se argumenta que legislaciones de igualdad de remuneración podrían comenzar a reducir estas brechas. También se recomiendan políticas dirigidas a promover la igualdad en el hogar mediante, por ejemplo, la enseñanza de los beneficios del empleo formal.

(c) 2015 Asociación Cuadernos de Economía. Publicado por Elsevier España, S.L.U. Todos los derechos reservados.
\end{abstract}

\section{Is there discrimination against women in the Ecuadorian labour market?}

Abstract Most studies addressing gender inequality focus exclusively on the wage gap. This work aims at correcting this bias by focusing on factors such as employment, formality of employment and underemployment. Ecuador has been chosen as a scenario for this study because of its relatively unique labour market structure in Latin America. Using survey data at a household level, this study finds evidence of discrimination against women in the form of lower wages and disparities in terms of employment, underemployment and formality. It is argued that equal pay legislations could foster the reduction of these gaps. This study also recommends policies driven to promote equality at home by, for example, teaching the benefits of formal employment to household members.

(c) 2015 Asociación Cuadernos de Economía. Published by Elsevier España, S.L.U. All rights reserved. 


\section{Introducción}

La desigualdad es una característica notable de América Latina: la región sigue siendo significativamente más desigual que Asia, que África, que Europa del Este y que la OCDE (Weller, 2012). El coeficiente de GINI promedio en América Latina, por ejemplo, es de 48 frente a 31 en los países de altos ingresos, 40 en Asia y 43 en África (UNCTAD, 2012). Sin embargo, en el inicio del nuevo milenio la desigualdad de ingresos se redujo de forma inequívoca en la mayoría de países de América Latina (Lustig, Lopez-Calva y Ortiz-Juarez, 2013). El coeficiente de GINI, por ejemplo, se redujo en un $1,3 \%$ en la región desde el año 2000 (Ortiz y Cummins, 2011) ${ }^{1}$. Esta mejora en la desigualdad se acompañó de una marcada disminución en la desigualdad de género en los últimos años. Por ejemplo, el índice de desigualdad de género del PNUD, una medida compuesta de las diferencias de género en salud reproductiva, empoderamiento y mercado laboral, disminuyó en la región desde el 0,47 al 0,43 en los 10 años previos a 2012. Este último se atribuye a factores como cambios en las tasas de fertilidad, la urbanización y mejoras en la infraestructura, que condujeron a una mayor participación por parte de las mujeres en el empleo formal (UNESCO, 2002). Además, Papadópulos y Radakovich (2006) sugieren que las recientes mejoras en la reducción de las diferencias de género en la región se deben en gran parte a un mayor acceso a la educación.

Sin embargo, en muchos países de la región la brecha salarial de género y la discriminación del mercado laboral siguen siendo un problema significativo (Paes de Barros, 2009). Además, permanecen en la región prácticas culturales machistas que dan ventajas significativas a los hombres sobre las mujeres (Deere y León, 2003; Abramo, 2004). Pautassi, Faur y Gherardi (2005), por ejemplo, argumentan que estereotipos socioculturales influenciaron a las leyes del mercado laboral en la región antes de las reformas neoliberales de la década de 1990. Por ejemplo, las mujeres fueron «protegidas» de la exposición a trabajos peligrosos, como aquellos que requieren turnos nocturnos; estos generaron impedimentos institucionales para la equidad laboral. A raíz de las reformas neoliberales estas leyes fueron cambiando; sin embargo, los paradigmas socioculturales se mantienen. Por lo tanto, existe hoy en día en Latinoamérica una estructura social que refuerza de manera informal las desigualdades de género, las cuales, por manera directa, entonces, refuerzan brechas salariales.

La persistencia de las diferencias salariales de género en América Latina ha sido explicada usando una variedad de hipótesis que toma en cuenta factores como la educación, la regulación gubernamental, las diferencias entre las zonas urbanas y rurales, las diferencias entre los sectores públicos y privados, trabajadores por cuenta propia, entre otras explicaciones (Atal, Nopo y Winder, 2009). En la mayoría de los casos la educación puede explicar la mayor parte de la brecha salarial de género, aunque en algunos casos la diferencia puede atribuirse a algún tipo de discriminación. Por ejemplo, Tenjo et al. (2005) sostienen que las diferencias

\footnotetext{
1 Lustig et al. (2013) argumentan que la disminución en la desigualdad se debe a transferencias gubernamentales, incrementos al salario mínimo y a la sindicalización, entre otros factores.
}

salariales se deben a las estructuras sociales tradicionales en las comunidades latinoamericanas que impiden que las mujeres amplíen sus oportunidades en los mercados laborales (Śniadecka-Kotarska y Gil Canova, 2006).

Además de examinar las diferencias salariales entre hombres y mujeres en América Latina, la mayoría de estudios han descuidado el análisis de otras características importantes en los mercados laborales. Como se señaló en García-Aracil y Winter (2006), esto es problemático porque los estudios de las brechas salariales excluyen a sujetos que están desempleados, subempleados o que trabajan por cuenta propia en el momento de la observación. Si esta exclusión afectara a los hombres y mujeres al azar no sería un problema, pero debido a que la participación en el mercado laboral es mayor para los hombres, es posible que aquellos estudios demuestren sesgos importantes. Este trabajo tiene como objetivo corregir este sesgo al enfocarse no solamente en diferencias salariales, sino también estudiando factores como el desempleo, la formalidad y el subempleo con el fin de investigar si las mujeres les va peor en función de otros indicadores importantes del mercado de trabajo. Esto es particularmente importante ya que, por ejemplo, la informalidad es más frecuente con las mujeres que con los hombres en algunos países de América Latina, incluyendo Ecuador (Arizpe, 1977; Marcouiller, Ruiz de Castilla y Woodruff, 1997; Calla y Milosavljevi, 2007). Además, la USAID-MTE, 2008 también encuentran evidencia de que existen diferencias persistentes de desempleo entre hombres y mujeres en Ecuador.

Podría decirse, sin embargo, que las mejoras en la equidad de género son más evidentes en Ecuador que en otros países de la región; allí, el índice de desigualdad de género disminuyó en un $14 \%$, en comparación con un $9 \%$ en la región, entre los años 2000 y 2012. Sin embargo, como en el resto de América Latina, las disparidades de género siguen siendo altas según estándares internacionales. Por otra parte, estructuras socioculturales machistas siguen predominando en la sociedad ecuatoriana (Abramo, 2004; UNESCO, 2002). Esto se ha convertido en una cuestión política apremiante en Ecuador, ya que el país continúa adoptando un estilo bolivariano de gobierno que tiene como objetivo hacer frente a las desigualdades sociales, incluyendo diferencias injustas entre los géneros (Posso Serrano y Posso, 2009).

Estas diferencias de género se pueden manifestar dentro de mercados laborales como brechas salariales, mayor desempleo por parte de las mujeres, mayores tasas de subempleo o empleo informal. En algunos casos las diferencias se deben a que, por ejemplo, las mujeres elijen trabajar en sectores informales con menores sueldos, y en otros casos se deben a que las mujeres no tienen opción (Heckman y Pagés, 2003). Es el segundo caso el que suele preocupar a los estados, ya que constituye un grado importante y significante de discriminación de género. Hasta donde sabemos solo hay 2 estudios económicos que han tratado de explicar las diferencias laborales entre hombres y mujeres en Ecuador. Estos estudios se han centrado únicamente en la brecha salarial de género. García-Aracil y Winter (2006) concluyen que muy poco de la diferencia en la brecha salarial de género se debe al nivel de instrucción, mientras que la mitad se puede atribuir a la discriminación. Por otra parte, Larrea y Montenegro Torres (2006) encuentran que el 74\% de la brecha salarial se debe a diferencias en la educación, mientras que el $26 \%$ puede atribuirse a discriminación en el mercado 
laboral. Aparte de que estos estudios se han preocupado únicamente con brechas salariales, también no han tomado en consideración el posible problema de sesgo de selección debido a la decisión de algunas personas de no participar en el mercado laboral. Esto se puede hacer mediante un corrección de Heckman, algo que se ha convertido en una práctica habitual en la literatura (Neuman y Oaxaca, 2004) ${ }^{2}$. En el presente estudio, se llevan a cabo 2 tipos de ajustes Heckman, tomando en cuenta si el nivel de instrucción y otros importantes predictores de éxito en el mercado laboral son responsables de la brecha salarial de género.

\section{El mercado laboral ecuatoriano en un contexto latinoamericano}

Ecuador comparte una serie de características importantes con la mayoría de países latinoamericanos; esto hace del mercado laboral ecuatoriano un estudio de caso particularmente apto para la región. Por ejemplo, desde el año 2000 se ha producido un modesto crecimiento del PIB junto con una alta generación de empleo (Pagés et al., 2009). Por otra parte, la mayoría de este trabajo se ha generado en sectores de baja productividad relativa (Weller, 2011). Además, ha habido un cambio político significativo hacia la izquierda en los últimos años (Madrid, 2010). Sin embargo, Ecuador se diferencia de otros países de América Latina en que, en general, su mercado laboral mantiene características más consistentes con un paradigma laissez-faire o neoliberal (Cano, 2010). En general, la flexibilidad del mercado laboral de Ecuador sugiere que es menos probable que diferencias laborales, en términos de género, se deban a limitaciones institucionales, y es más probable que se deban a factores socioculturales.

Hay al menos 2 razones claves por las que el mercado laboral ecuatoriano se asemeja a un mercado de trabajo neoclásico. En primer lugar, la debilidad de las instituciones significa que el incumplimiento con las leyes laborales va a ser más común (Maclsaac y Rama, 1997; León, 2012). Esto se debe a la debilidad de la capacidad de ejecución, los tribunales saturados y la presencia de funcionarios corruptos que han encontrado maneras de adquirir rentas a cambio de ayudar a algunas empresas a evitar los costos asociados con algunas leyes laborales (Maclsaac y Rama, 1997; Basabe-Serrano, 2013). En segundo lugar, las leyes que tienen por objeto proteger a los trabajadores en el Ecuador están plagadas de ambigüedades que las hacen sumamente ineficaces. Por ejemplo, en el nuevo milenio, los gobiernos de Gustavo Noboa (2000-2002) y Lucio Gutiérrez (2002-2005) se embarcaron en reformas del mercado laboral mediante la emisión de las llamadas leyes Trole, que tienen por objeto proteger a los trabajadores y regular el mercado. Sin embargo, muchos aspectos del código laboral solo son aplicables a determinadas industrias y a ciertas partes del sector público. Por lo tanto, mientras que algunos trabajadores tenían el derecho a hacer huelga, a la negociación

\footnotetext{
${ }^{2}$ Esto es problemático ya que la participación masculina en mercados laborales es mayor, lo que quiere decir que las mujeres tienen más probabilidades de ser excluidas de ecuaciones que se concentran únicamente en los ingresos.
}

colectiva y a la sindicalización, las firmas dedicadas a la fabricación, servicios, y los sectores militares, en general, no tuvieron este tipo de protección (Cano, 2010). Esto significa que el código laboral no protege a una gran parte de los trabajadores ecuatorianos.

El año 2007 vio la elección de Rafael Correa como presidente; este prometió una serie de reformas encaminadas a reducir la desigualdad y la pobreza. Estas reformas fueron encabezadas por los cambios en la Constitución del 2008 y una nueva ley laboral: el Mandato Presidencial N. ${ }^{\circ} 8$ de ese mismo año. Curiosamente, sin embargo, las ambigüedades de los decretos pasados se mantuvieron, sin dar lugar a algún cambio real en la situación del mercado laboral. Por ejemplo, el artículo 229 de la Constitución establece que los trabajadores están cubiertos por el Código de Trabajo. Sin embargo, el artículo 326 del mismo excluye a los trabajadores que realizan tareas administrativas o profesionales, es decir, la mano de obra calificada (Cano, 2010). Excluyendo a los trabajadores calificados de las leyes laborales en un país como Ecuador hace que el código sea casi totalmente ineficaz, porque una gran proporción de trabajadores no calificados trabajan en el sector informal, donde las leyes no pueden aplicarse (Pagés et al., 2009; Weller, 2011).

Del mismo modo, el Mandato $\mathrm{N}{ }^{\circ} 8$, que tenía por objeto la eliminación de la tercerización y otras formas de contratación de la mano de obra precaria, en un esfuerzo por acabar con los mercados de trabajo informales, en gran parte ha sido una medida ineficaz (Salazar Maldonado, 2009). Esta ley abolió la subcontratación, contratación por horas y cualquier forma de precariedad laboral. Sin embargo, la ley también establece que los trabajadores solo pueden apelar a estas normas dentro del primer año de trabajo en una empresa. Por otra parte, las empresas pueden decidir ignorar este mandato si se enfrentan a la escasez de mano de obra, debido a la inexperiencia técnica, actividades complementarias o la necesidad de un trabajo a tiempo parcial (Cano, 2010). Por último, los trabajadores solo tienen derecho a ser representados por la negociación colectiva después de su segundo año de empleo, y esto solo se aplica a la definición anteriormente implícita de los trabajadores (no calificados).

No es de extrañar, pues, que de acuerdo con Salazar Maldonado (2009) la informalidad mantiene su tendencia, que abarca una gran proporción de la población económicamente activa. De hecho, un informe en el 2013 por el Instituto Nacional de Estadísticas y Censos (INEC) indica que el sector informal abarca el $40 \%$ de la población activa total en 5 grandes centros urbanos del Ecuador ${ }^{3}$. Esto constituye una mejora de únicamente 5 puntos porcentuales desde el inicio de Mandato N. ${ }^{\circ}$. Además, ese mismo informe estima que el $47 \%$ de la población puede ser categorizada como subempleada, a partir de marzo del 2013.

Por último, y tal vez irónicamente, la ideología anticorporativa del gobierno de Correa también ha contribuido activamente a la ineficacia general de las iniciativas laborales. El gobierno de Ecuador, por ejemplo, se ha opuesto activamente a la negociación colectiva, que ha sido calificada como un legado del «corporativismo», basada en

\footnotetext{
${ }^{3}$ La encuesta de INEC cubre las ciudades más grandes del país: Quito, Guayaquil, Cuenca, Machala y Ambato.
} 
ideologías capitalistas norteamericanas. Como resultado, la mayoría de la legislación de la Asamblea Nacional ha buscado limitar los sindicatos públicos, impedir la sindicalización de los empleados y reducir al mínimo cualquier posibilidad de negociación colectiva. Además, poco después de pasar el Mandato $\mathrm{N} .{ }^{\circ} 8$, el gobierno se trasladó a eliminar las contribuciones estatales a los fondos privados de pensiones e indemnizaciones por despido de los trabajadores del sector público (Ospina Peralta, 2011).

En general, la ineficacia o falta de voluntad del gobierno para hacer cumplir las leyes laborales, junto con las instituciones persistentemente débiles, sugiere que el mercado laboral ecuatoriano sigue siendo relativamente libre. Por lo tanto, es menos probable que las diferencias de género dentro de este mercado se derivan de factores institucionales, y es más probable que se deban a imperantes factores socioculturales.

\section{Datos}

Este análisis utiliza datos de la Encuesta de Condiciones de Vida, Quinta Ronda ECV (INEC, 2006). La encuesta nacional se llevó a cabo por el INEC a partir de octubre del 2005 a septiembre del 2006. Este estudio utiliza estos datos por 3 razones. En primer lugar, a pesar de que el INEC administró encuestas en el 2010 y 2012, su cobertura de los resultados del mercado laboral no fue tan amplia como la encuesta del 2005-2006. Por ejemplo, las encuestas del 2010 y 2012 no realizaron preguntas sobre contratos laborales y la frecuencia de empleo. Además, la oficina estadística ecuatoriana no ha hecho públicos todos los datos necesarios, como características del hogar y niveles de educación de los padres, para un análisis econométrico. En segundo lugar, como se ha señalado por la OIT (2011) y Dávalos (2012), la crisis económica de 2008-2011 tuvo consecuencias adversas en el mercado laboral del Ecuador, principalmente por el hecho de que Ecuador es una economía abierta dolarizada dependiente de las exportaciones de materias primas. Por lo tanto, es poco probable que el censo más reciente dé una indicación fiable de las condiciones laborales ecuatorianas a largo plazo. El período de 2005-2006, por lo contrario, fue un período de relativa estabilidad económica (Pagés et al., 2009). En tercer lugar, como se mencionó en la sección número 2 , a pesar de que la encuesta se llevó a cabo 2 años antes de una serie de reformas legales, existe evidencia de que estos datos aún representan las condiciones de los trabajadores hoy en día. Por ejemplo, usando datos salariales de la encuesta realizada en 2012 se puede observar que existía en ese entonces una brecha de género parecida a la que se puede ver en el 2005 y 2006. La figura A1, en el Apéndice 1, demuestra que en el 2012 existía una brecha salarial entre hombres y mujeres (de 15 a 65 años) del $54 \%$, mientras que la figura 1 (abajo) demuestra que esta brecha en el 2006 era del 60\%. Entonces, podemos concluir que las brechas de género en los mercados laborales ecuatorianos parecen moverse lentamente. Esto es consistente con la evidencia internacional que demuestra que los cambios en los mercados laborales son lentos (véase Blau, Brummund y Liu, 2013). Dicho esto, es importante ser cauto con los resultados obtenidos, ya que no podemos, necesariamente, extrapolarlos a la situación actual. Es decir, el análisis demuestra una situación laboral evidente en los años 2005-2006 que puede ayudar a informar sobre la situación actual, aunque las brechas que observamos pueden haber cambiado marginalmente.

La Encuesta de Condiciones de Vida encuestó a 55.666 personas, de las cuales 13.581 eran cabeza de hogar y 8.347 eran sus respectivos cónyuges. El análisis econométrico se concentra en estos individuos, aunque las ecuaciones estimadas varían en función de la disponibilidad y frecuencia de las respuestas. Las variables dependientes
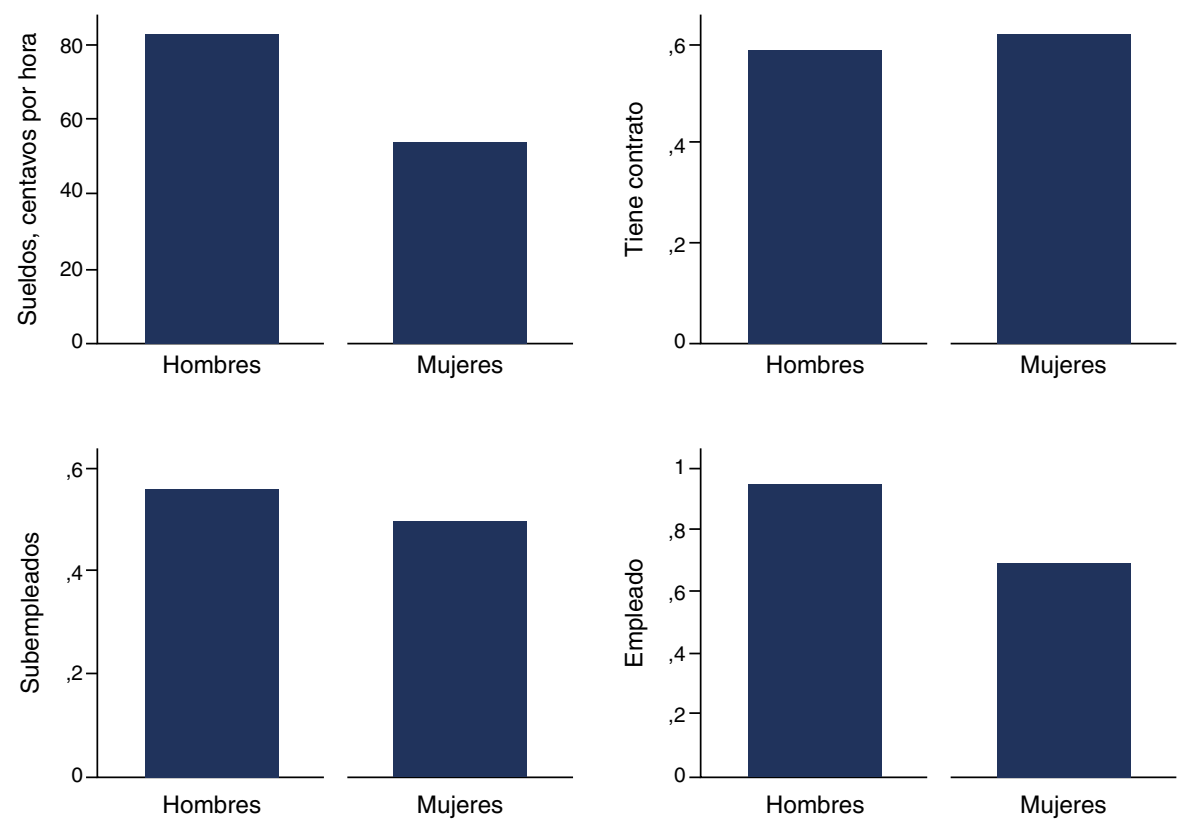

Figura 1 Condiciones laborales generales en Ecuador, por género.

Fuente: Encuesta de Condiciones de Vida (2005-2006). 
Tabla 1 Estadísticas descriptivas

\begin{tabular}{|c|c|c|c|c|c|}
\hline Variable & Obs & Signif. & $\mathrm{DE}$ & Min & Max \\
\hline Salarios por hora & 1531 & 79,38 & 40,89 & 2,10 & 833,33 \\
\hline Subempleados & 1092 & 0,43 & 0,50 & 0,00 & 1,00 \\
\hline Empleado & 7294 & 0,64 & 0,48 & 0,00 & 1,00 \\
\hline Tiene contrato & 1154 & 0,63 & 0,48 & 0,00 & 1,00 \\
\hline Mujer & 7294 & 0,53 & 0,49 & 0,00 & 1,00 \\
\hline Indígena & 7294 & 0,08 & 0,27 & 0,00 & 1,00 \\
\hline Edad & 7294 & 40,81 & 14,14 & 14,00 & 90,00 \\
\hline Tenencia & 7294 & 13,11 & 14,22 & 0,00 & 74,00 \\
\hline Educación & 7294 & 5,07 & 1,88 & 1,00 & 9,00 \\
\hline Casado/a & 7294 & 0,68 & 0,47 & 0,00 & 1,00 \\
\hline Estudios del padre & 7294 & 2,21 & 1,71 & 1,00 & 5,00 \\
\hline Estudio de la madre & 7294 & 2,40 & 1,80 & 1,00 & 5,00 \\
\hline Enfermo & 7294 & 0,62 & 0,49 & 0,00 & 1,00 \\
\hline Migrantes recientes & 7294 & 0,08 & 0,27 & 0,00 & 1,00 \\
\hline Niños & 7294 & 2,96 & 1,83 & 0,00 & 19,00 \\
\hline
\end{tabular}

Fuente: Encuesta de Condiciones de Vida (2005-2006).

son: salarios (medidos en centavos de dólar por hora), subempleados (una variable igual a 1 si el encuestado está dispuesto a trabajar más horas), empleado (una variable igual a 1 si el encuestado está empleado) y contrato (un variable igual a 1 si el encuestado tiene un contrato).

La variable «contrato» se utiliza como sustituta de informalidad de empleo. Hay 2 definiciones de informalidad ampliamente utilizadas. La definición clásica categoriza como empleo informal a uno que cae fuera del sector industrial moderno (Lewis, 2013). La segunda definición, que se utiliza en este documento, clasifica a los trabajadores informales como aquellos que no tienen un contrato que garantiza la seguridad de empleo, la seguridad social y las prestaciones (como el pago de horas extras, asistencia en caso de pérdida de empleo, y anual de licencia derechos). En general, la informalidad se caracteriza por las condiciones irregulares de trabajo, alta rotación y menores tasas de remuneración. Esta definición se aplica ampliamente en América Latina (Maloney, 2004; Henley, Arabsheibani y Carneiro, 2009).

La figura 1 resume las variables dependientes, tanto para hombres y mujeres, en las 21 provincias del Ecuador. Los datos muestran que, en promedio, las mujeres ganan menos por hora que los hombres y tienen menos probabilidades de ser empleadas. Por el otro lado, más hombres se encuentran subempleados y trabajando informalmente. El salario por hora para los hombres y las mujeres representadas en la figura parece bajo; sin embargo, es más o menos consistente con la tarifa por hora del salario de los trabajadores de producción en Ecuador (1988-2008) medido en el 2006 según las estimaciones de datos salariales de la UNIDO.

Los regresores utilizados para explicar las 4 variables dependientes tienen como objetivo cubrir los determinantes generales de los resultados del mercado laboral. La variable «mujer» se incluye para capturar las diferencias de género. Tenencia, la edad y el nivel de educación (de ninguno a posgrado) también son utilizadas. Tenencia se mide por el número de años en el trabajo actual, mientras que la educación es una escala ordinal de autorreporte que va desde ninguno (1) a posgrado (9) ${ }^{4}$. Una variable dummy igual a 1 se incluye para identificar si los encuestados se autoidentifican como indígenas con el fin de capturar cualquier tipo de discriminación racial en los mercados laborales ${ }^{5}$. Siguiendo a Chun y Lee (2001), los modelos incluyen una variable binaria igual a 1 si el/la entrevistado/a está casado/a, mientras que siguiendo a Altonji y Dunn (1996) los modelos también incluyen los niveles de educación del padre y de la madre del entrevistado. El análisis de regresión también incluye una variable binaria igual a uno si el entrevistado señaló que él o ella estaba enfermo en el último mes. Curiosamente, aproximadamente el $60 \%$ de los encuestados reportaron estar «enfermos», lo que sugiere que esta variable puede capturar cualquier pequeña enfermedad, incluyendo resfriados o gripes. Una variable de migración se incluye para capturar cualquier forma de prejuicio contra los pueblos de comunidades regionales: la variable «migrante reciente» es igual a 1 si el entrevistado se mudó a su ubicación actual en los últimos 5 años. Por otra parte, también se incluye el número total de hijos a cargo de los encuestados. La tabla 1 resume el resto de datos.

\section{Metodología}

Tradicionalmente, la forma más común para analizar la discriminación por género es comparando los ingresos, por ejemplo, que reciben los hombres y las mujeres, manteniendo constante la productividad (Oaxaca, 1973; Blinder, 1973; Oaxaca y Ransom, 1994). Un método es simplemente incluir un dummy de género en los modelos de regresión, para estimar la siguiente ecuación:

$R M T=\alpha+\lambda Z+\beta F+\varepsilon$,

\footnotetext{
${ }^{4}$ Términos al cuadrado de la tenencia y la edad también se incluyeron en las regresiones; sin embargo, estos resultaron ser estadísticamente insignificantes.

${ }^{5}$ Véase, por ejemplo, García-Aracil y Winter (2006) y Calla y Milosavljevi (2007).
} 
Tabla 2 Discriminación contra la mujer: MCO y modelos Probit

\begin{tabular}{|c|c|c|c|c|}
\hline Variables & $\begin{array}{l}\text { (1) } \\
\text { Salarios por hora }\end{array}$ & $\begin{array}{l}(2) \\
\text { Subempleados }\end{array}$ & $\begin{array}{l}\text { (3) } \\
\text { Empleado }\end{array}$ & $\begin{array}{l}(4) \\
\text { Tiene contrato }\end{array}$ \\
\hline Mujer & $\begin{array}{l}-14,6^{* * *} \\
{[-4,29]}\end{array}$ & $\begin{array}{l}-0,067 \\
{[-1,83]}\end{array}$ & $\begin{array}{l}-0,40^{\text {*** }} \\
{[-5,33]}\end{array}$ & $\begin{array}{l}-0,020 \\
{[-1,26]}\end{array}$ \\
\hline Indígena & $\begin{array}{l}1,42 \\
{[0,36]}\end{array}$ & $\begin{array}{l}-0,10^{\star * \star} \\
{[-3,39]}\end{array}$ & $\begin{array}{l}0,25^{* * *} \\
{[6,40]}\end{array}$ & $\begin{array}{l}-0,014 \\
{[-0,85]}\end{array}$ \\
\hline Edad & $\begin{array}{l}-0,080 \\
{[-0,74]}\end{array}$ & $\begin{array}{l}-0,0062 \\
{[-0,16]}\end{array}$ & $\begin{array}{l}-0,0020 \\
{[-0,30]}\end{array}$ & $\begin{array}{l}0,00014 \\
{[0,022]}\end{array}$ \\
\hline Tenencia & $\begin{array}{l}0,19^{*} \\
{[1,82]}\end{array}$ & $\begin{array}{l}-0,0017 \\
{[-0,16]}\end{array}$ & & $\begin{array}{l}-0,000046 \\
{[-0,022]}\end{array}$ \\
\hline Educación & $\begin{array}{l}2,24^{* *} \\
{[2,46]}\end{array}$ & $\begin{array}{l}0,026 \\
{[0,16]}\end{array}$ & $\begin{array}{l}0,045 \\
{[0,30]}\end{array}$ & $\begin{array}{l}0,017 \\
{[0,022]}\end{array}$ \\
\hline Casado/a & $\begin{array}{l}-0,68 \\
{[-0,27]}\end{array}$ & $\begin{array}{l}0,011 \\
{[0,60]}\end{array}$ & $\begin{array}{l}0,029^{*} \\
{[1,76]}\end{array}$ & $\begin{array}{l}0,017 \\
{[1,25]}\end{array}$ \\
\hline Estudio del padre & $\begin{array}{l}-0,031 \\
{[-0,048]}\end{array}$ & $\begin{array}{l}-0,011 \\
{[-0,16]}\end{array}$ & $\begin{array}{l}0,0036 \\
{[0,28]}\end{array}$ & $\begin{array}{l}0,0011 \\
{[0,022]}\end{array}$ \\
\hline Estudio de la madre & $\begin{array}{l}0,30 \\
{[0,45]}\end{array}$ & $\begin{array}{l}0,0091 \\
{[0,16]}\end{array}$ & $\begin{array}{l}0,010 \\
{[0,30]}\end{array}$ & $\begin{array}{l}0,00042 \\
{[0,022]}\end{array}$ \\
\hline Enfermo/a & $\begin{array}{l}-4,17^{* *} \\
{[-1,97]}\end{array}$ & $\begin{array}{l}0,052^{* * *} \\
{[3,50]}\end{array}$ & $\begin{array}{l}0,037^{* * *} \\
{[2,68]}\end{array}$ & $\begin{array}{l}-0,0019 \\
{[-0,53]}\end{array}$ \\
\hline Migrantes recientes & $\begin{array}{l}-5,21 \\
{[-1,21]}\end{array}$ & $\begin{array}{l}0,015 \\
{[0,54]}\end{array}$ & $\begin{array}{l}-0,11^{\text {*** }} \\
{[-4,61]}\end{array}$ & $\begin{array}{l}-0,0013 \\
{[-0,23]}\end{array}$ \\
\hline Niños & $\begin{array}{l}0,85 \\
{[1,61]}\end{array}$ & $\begin{array}{l}-0,0010 \\
{[-0,14]}\end{array}$ & $\begin{array}{l}0,021 \\
{[0,30]}\end{array}$ & $\begin{array}{l}-0,0011 \\
{[-0,022]}\end{array}$ \\
\hline Efectos fijos: ¿ocupación? & Sí & Sí & No & Sí \\
\hline Efectos fijos: ¿municipio? & Sí & Sí & Sí & Sí \\
\hline Observaciones & 1531 & 1092 & 7294 & 4173 \\
\hline R-cuadrado & 0,44 & & & \\
\hline P-valor al cuadrado-Chi & & 0 & 0 & 0 \\
\hline
\end{tabular}

Estadísticas t entre paréntesis. La columna 1 utiliza MCO. Las columnas 2, 3 y 4 muestran los efectos marginales de las regresiones Probit.

", "*, $\mathrm{y}{ }^{* * *}$ denotan niveles del 10,5 y $1 \%$ de significancia, respectivamente.

donde RMT es un resultado del mercado de trabajo, como salarios, $Z$ es un vector de variables explicativas, como la educación, $F$ es una variable binaria igual a uno si el sujeto es mujer, y $\varepsilon$ es un término de error idiosincrásico (Kim y Polachek, 1994). Este enfoque se adoptó en la tabla 2, donde los salarios por hora se analizaron mediante mínimos cuadrados ordinarios (MCO), mientras que el resto de variables se analizaron mediante modelos Probit porque las variables dependientes son binarias.

El principal problema de la estimación de la ecuación (1) es que la magnitud de $\beta$ puede ser debida a la discriminación o una serie de otros factores, los cuales pueden estar sujetos a la interpretación del investigador. Por ejemplo, aunque las mujeres tienen el mismo nivel de educación que los hombres, los hombres podrían enfocar sus estudios en la consecución de habilidades que reciben mayores recompensas en los mercados laborales; por lo tanto, la preparación educativa se cargará en la variable $F$, enturbiando la interpretación como discriminación (Oaxaca, 1973; Blinder, 1973; Oaxaca y Ransom, 1994).

Para hacer frente a este problema, las tablas 3-5 se basan en un método propuesto por Blinder (1973) y Oaxaca (1973), que toman en cuenta que al menos una parte de la diferencia entre hombres y mujeres en los resultados del mercado laboral no se debe a la discriminación, sino al hecho de que los 2 grupos tienen, en promedio, características diferentes
(Altonji y Blank, 1999). En este caso, los resultados del mercado de trabajo se estiman separado cada género usando las variables de control que se resumen en la sección anterior. Entonces, estas ecuaciones se utilizan para estimar un diferencial $(R)$, como se especifica en la siguiente ecuación:

$R=E\left(R M T_{M}\right)-E\left(R M T_{F}\right)$

donde $E\left(R M T_{i}\right)$ denota el valor esperado de la variable de resultado para el grupo $i$, donde a su vez $i$ es ya sea hombres $(M)$ o mujeres $(F)$. Blinder (1973) y Oaxaca (1973) proponen una descomposición doble de $R$ en los componentes $Q$ y $U$. El primer componente, $Q$, se explica por diferencias de grupo determinadas por los predictores (la «cantidad» o efecto explicado), mientras que $U$ se refiere a la parte «inexplicable». Este último se atribuye a la discriminación.

La determinación de $Q$ y $U$ requiere una interpretación adecuada de las estimaciones de los coeficientes del vector de variables no discriminatorias, es decir, la estimación del coeficiente unido al vector de variables que componen a $Q$. Esto no es trivial, ya que puede cambiar la forma en la que la ecuación (2) se estima. Por ejemplo, si se supone que la discriminación se dirige únicamente en contra de las mujeres y no a favor de los hombres, entonces la ecuación (2) se estima utilizando coeficientes femeninos como coeficientes de referencia; por otra parte, si suponemos que solo hay discriminación positiva a favor de los hombres, 
Tabla 3 Descomposición Blinder-Oaxaca: los hombres frente a mujeres en el mercado laboral ecuatoriano

\begin{tabular}{|c|c|c|c|c|}
\hline Var Dep: & $\begin{array}{l}(1) \\
\text { Salarios por hora }\end{array}$ & $\begin{array}{l}(2) \\
\text { Subempleados }\end{array}$ & $\begin{array}{l}(3) \\
\text { Empleado }\end{array}$ & $\begin{array}{l}(4) \\
\text { Tiene contrato }\end{array}$ \\
\hline \multicolumn{5}{|l|}{ Panel $A$} \\
\hline Predicción hombre & $\begin{array}{l}83,1^{* * *} \\
{[73,4]}\end{array}$ & $\begin{array}{l}0,54^{* * *} \\
{[34,9]}\end{array}$ & $\begin{array}{l}0,93^{* * *} \\
{[66,3]}\end{array}$ & $\begin{array}{l}0,58^{* * *} \\
{[58,6]}\end{array}$ \\
\hline Predicción mujer & $\begin{array}{l}54,3^{* * * *} \\
{[28,8]}\end{array}$ & $\begin{array}{l}0,54^{*+* *} \\
{[11,0]}\end{array}$ & $\begin{array}{l}0,63^{* * *} \\
{[109]}\end{array}$ & $\begin{array}{l}0,62^{* * *} \\
{[46,4]}\end{array}$ \\
\hline Diferencia & $\begin{array}{l}28,9^{* * *} \\
{[13,1]}\end{array}$ & $\begin{array}{l}0,0045 \\
{[0,087]}\end{array}$ & $\begin{array}{l}0,30^{* * *} \\
{[19,9]}\end{array}$ & $\begin{array}{l}-0,035^{* *} \\
{[-2,11]}\end{array}$ \\
\hline \multicolumn{5}{|l|}{ Panel B: descomposición } \\
\hline Explicado (Q) & $\begin{array}{l}14,3^{* * *} \\
{[6,50]}\end{array}$ & $\begin{array}{l}0,0054 \\
{[0,099]}\end{array}$ & $\begin{array}{l}-0,0019 \\
{[-0,31]}\end{array}$ & $\begin{array}{l}-0,096^{\text {*** }} \\
{[-7,84]}\end{array}$ \\
\hline Inexplicable $(U)$ & $\begin{array}{l}14,6^{* * *} \\
{[7,33]}\end{array}$ & $\begin{array}{l}-0,00094 \\
{[-0,014]}\end{array}$ & $\begin{array}{l}0,30^{* * *} \\
{[20,9]}\end{array}$ & $\begin{array}{l}0,060^{* * *} \\
{[4,80]}\end{array}$ \\
\hline \multicolumn{5}{|c|}{ Panel C: descomposición del efecto explicado } \\
\hline Indígena & $\begin{array}{l}-0,11 \\
{[-0,49]}\end{array}$ & $\begin{array}{l}0,0054 \\
{[0,63]}\end{array}$ & $\begin{array}{l}0,0012 \\
{[0,41]}\end{array}$ & $\begin{array}{l}-0,00093 \\
{[-1,28]}\end{array}$ \\
\hline Tenencia & $\begin{array}{l}-0,18 \\
{[-0,76]}\end{array}$ & $\begin{array}{l}-0,00032 \\
{[-0,12]}\end{array}$ & & $\begin{array}{l}-0,000048 \\
{[-0,21]}\end{array}$ \\
\hline Edad & $\begin{array}{l}-0,075 \\
{[-0,58]}\end{array}$ & $\begin{array}{l}-0,0028 \\
{[-0,29]}\end{array}$ & $\begin{array}{l}-0,00019 \\
{[-0,36]}\end{array}$ & $\begin{array}{l}0,00013 \\
{[0,52]}\end{array}$ \\
\hline Educación & $\begin{array}{l}0,75^{* *} \\
{[1,76]}\end{array}$ & $\begin{array}{l}0,021 \\
{[0,77]}\end{array}$ & $\begin{array}{l}-0,0016 \\
{[-0,39]}\end{array}$ & $\begin{array}{l}-0,040^{* * *} \\
{[-9,34]}\end{array}$ \\
\hline Casado & $\begin{array}{l}0,045 \\
{[0,29]}\end{array}$ & $\begin{array}{l}-0,0023 \\
{[-0,42]}\end{array}$ & $\begin{array}{l}-0,00015 \\
{[-0,36]}\end{array}$ & $\begin{array}{l}0,0044^{* * *} \\
{[3,30]}\end{array}$ \\
\hline Estudio padre & $\begin{array}{l}-0,00095 \\
{[-0,049]}\end{array}$ & $\begin{array}{l}-1.3 e-06 \\
{[-0,013]}\end{array}$ & $\begin{array}{l}0,00011 \\
{[0,36]}\end{array}$ & $\begin{array}{l}-0,000026 \\
{[-0,14]}\end{array}$ \\
\hline Estudio de la madre & $\begin{array}{l}-0,094 \\
{[-0,52]}\end{array}$ & $\begin{array}{l}-0,0053 \\
{[-0,65]}\end{array}$ & $\begin{array}{l}0,00044 \\
{[0,39]}\end{array}$ & $\begin{array}{l}0,00021 \\
{[0,34]}\end{array}$ \\
\hline Enfermo & $\begin{array}{l}0,48^{*} \\
{[1,78]}\end{array}$ & $\begin{array}{l}-0,0068 \\
{[-0,74]}\end{array}$ & $\begin{array}{l}-0,00032 \\
{[-0,38]}\end{array}$ & $\begin{array}{l}0,00090 \\
{[0,59]}\end{array}$ \\
\hline Reciente Mig & $\begin{array}{l}-0,050 \\
{[-0,49]}\end{array}$ & $\begin{array}{l}0,00044 \\
{[0,20]}\end{array}$ & $\begin{array}{l}6.4 e-07 \\
{[0,0043]}\end{array}$ & $\begin{array}{l}-0,00012 \\
{[-0,25]}\end{array}$ \\
\hline Niños & $\begin{array}{l}-0,51^{*} \\
{[-1,72]}\end{array}$ & $\begin{array}{l}0,0047 \\
{[0,63]}\end{array}$ & $\begin{array}{l}-0,000087 \\
{[-0,28]}\end{array}$ & $\begin{array}{l}-0,0015 \\
{[-1,22]}\end{array}$ \\
\hline Efectos fijos: ¿ocupación? & Sí & Sí & Sí & Sí \\
\hline Efectos fijos: ¿municipio? & Sí & Sí & No & Sí \\
\hline Observaciones & 1531 & 1092 & 7294 & 4173 \\
\hline
\end{tabular}

Estadísticas t entre paréntesis. La columna 1 utiliza MCO. Las columnas 2, 3 y 4 muestran los efectos marginales de las regresiones Probit.

${ }^{*},{ }^{* *}, \mathrm{y}^{* * *}$ denotan niveles del 10,5 y $1 \%$ de significancia, respectivamente.

entonces la ecuación (2) se calcula usando coeficientes masculinos como grupo de referencia. Este artículo sigue a Jann (2008) y supone que no hay ninguna razón específica para suponer que los coeficientes de ambos sexos son discriminatorios. Por lo tanto, la ecuación (2) se estima utilizando los coeficientes promedio de hombres y mujeres para la estimación del vector de parámetros no discriminatorios $(Q)^{6}$. En efecto, en la interpretación de las estimaciones de los coeficientes unidos a la descomposición, este método todavía crea un grupo de «control» (hombres) y mide las diferencias con un grupo «experimental» (mujeres).

\footnotetext{
${ }^{6}$ Como un ejercicio de robustez se intentaron diferentes pesos. Los resultados siguen siendo consistente a través de las distintas especificaciones.
}

Las regresiones se estiman en 2 etapas. En primer lugar, un modelo combinado sobre ambos grupos se estima utilizando MCO o Probit, dependiendo de la variable dependiente. En segundo lugar, el sistema calcula el grupo de medios de los predictores basados en el vector de parámetros y la matriz de varianza-covarianza combinada con los coeficientes de los modelos y de las estimaciones promedias. Se estiman los modelos Probit siguiendo el método propuesto por Yun (2004), que cuantifica la contribución de las diferencias entre los grupos a las variables independientes en la diferencia de resultados como efectos marginales ${ }^{7}$.

\footnotetext{
7 Una metodología alternativa es propuesta por Fairlie (1999, 2005), lo que da resultados consistentes a los previstos en las siguientes.
} 
Tabla 4 Descomposición Heckman Blinder-Oaxaca: los hombres frente a mujeres en el mercado laboral ecuatoriano

\begin{tabular}{|c|c|c|c|c|}
\hline Var Dep: & $\begin{array}{l}\text { (1) } \\
\text { Salarios por hora }\end{array}$ & $\begin{array}{l}(2) \\
\text { Subempleados }\end{array}$ & $\begin{array}{l}\text { (3) } \\
\text { Empleado }\end{array}$ & $\begin{array}{l}(4) \\
\text { Tiene contrato }\end{array}$ \\
\hline \multicolumn{5}{|l|}{ Panel $A$} \\
\hline \multirow[t]{2}{*}{ Predicción hombre } & $85,0^{* * *}$ & $0,58^{* * *}$ & $0,95^{* * *}$ & $0,60^{* * *}$ \\
\hline & {$[55,3]$} & {$[20,4]$} & {$[70,2]$} & {$[39,7]$} \\
\hline \multirow[t]{2}{*}{ Predicción mujer } & $54,3^{* * *}$ & $0,35^{* * *}$ & $0,58^{* * *}$ & $0,64^{* * *}$ \\
\hline & {$[28,8]$} & {$[3,58]$} & {$[50,3]$} & {$[28,9]$} \\
\hline \multirow[t]{2}{*}{ Diferencia } & $30,7^{* * *}$ & $0,23^{* *}$ & $0,37^{* \star *}$ & $-0,049^{*}$ \\
\hline & {$[12,6]$} & {$[2,21]$} & {$[20,9]$} & {$[-1,82]$} \\
\hline \multicolumn{5}{|l|}{ Panel B: descomposición } \\
\hline \multirow[t]{2}{*}{ Explicada $(Q)$} & $15,0^{* * *}$ & $-0,036$ & $-0,0029$ & $-0,093^{* * *}$ \\
\hline & {$[6,83]$} & {$[-0,67]$} & {$[-0,39]$} & {$[-7,63]$} \\
\hline \multirow[t]{2}{*}{ Inexplicable $(U)$} & $15,7^{* * *}$ & $0,26^{* *}$ & $0,37^{* * *}$ & $0,044^{*}$ \\
\hline & {$[6,84]$} & {$[2,32]$} & {$[21,0]$} & {$[1,79]$} \\
\hline Efectos fijos: ¿ocupación? & Sí & Sí & No & Sí \\
\hline Efectos fijos: ¿municipio? & Sí & Sí & Sí & Sí \\
\hline Observaciones & 1531 & 1092 & 7294 & 4173 \\
\hline
\end{tabular}

Estadísticas t entre paréntesis. Las columnas 1 y 3 utilizan MCO. Las columnas 2 y 4 muestran los efectos marginales de las regresiones Probit. Modelo 1 se refiere a la descomposición de sexo masculino, y el modelo 2 se refiere a la descomposición femenina.

", ", y ${ }^{* * *}$ denotan niveles del 10, 5 y $1 \%$ de significancia, respectivamente.

Como la mayoría de las técnicas en economía aplicada, la descomposición Blinder-Oaxaca ha recibido algunas críticas. Por ejemplo, Weichselbaumer y Winter-Ebmer (2005) argumentan que, en presencia de una variable omitida que se correlaciona positivamente con los componentes de $Q$, el efecto de discriminación será subestimado. Por otro lado, si las variables omitidas se correlacionan con el género, a continuación el residuo no solo capturará la discriminación, sino también las diferencias de grupo no observadas en la productividad. Un indicador de grupo (mujeres) se añade como una covariable en el modelo combinado para hacer frente a este problema; sin embargo, este método no es perfecto, lo que significa que los resultados aún deben ser interpretados con cautela (Jann, 2008).

Otra crítica importante de esta descomposición viene de Heckman (1979). Este autor sostiene que la brecha salarial de género y otras diferencias del mercado laboral existen porque los hombres y las mujeres tienen un conjunto diferente de opciones disponibles para ellos a la hora de participar en los mercados de trabajo. En este caso, tanto los componentes explicados e inexplicables pueden ser diferentes para cada grupo, lo que sugiere que los hombres son diferentes en formas observables y no observables a las mujeres en los mercados laborales, lo que quiere decir que

Tabla 5 Descomposición Heckman doble, Blinder-Oaxaca: los hombres frente a mujeres en el mercado laboral ecuatoriano

\begin{tabular}{|c|c|c|c|}
\hline Var Dep: & $\begin{array}{l}\text { (1) } \\
\text { Salarios por hora }\end{array}$ & $\begin{array}{l}\text { (2) } \\
\text { Subempleados }\end{array}$ & $\begin{array}{l}\text { (3) } \\
\text { Tiene contrato }\end{array}$ \\
\hline \multicolumn{4}{|l|}{ Panel $A$} \\
\hline Predicción hombre & $\begin{array}{l}84,9^{* * *} \\
{[55,5]}\end{array}$ & $\begin{array}{l}0,58^{* * *} \\
{[20,6]}\end{array}$ & $\begin{array}{l}0,60^{* * *} \\
{[39,8]}\end{array}$ \\
\hline Predicción mujer & $\begin{array}{l}54,3^{* * *} \\
{[28,8]}\end{array}$ & $\begin{array}{l}0,38^{* * * *} \\
{[3,52]}\end{array}$ & $\begin{array}{l}0,65^{* * *} \\
{[29,0]}\end{array}$ \\
\hline Diferencia & $\begin{array}{l}30,7^{* \star *} \\
{[12,6]}\end{array}$ & $\begin{array}{l}0,20^{*} \\
{[1,84]}\end{array}$ & $\begin{array}{l}-0,050^{*} \\
{[-1,88]}\end{array}$ \\
\hline \multicolumn{4}{|l|}{ Panel B: descomposición } \\
\hline Explicado $(Q)$ & $\begin{array}{l}14,9^{* * *} \\
{[6,79]}\end{array}$ & $\begin{array}{l}-0,044 \\
{[-0,82]}\end{array}$ & $\begin{array}{l}-0,092^{\text {****}} \\
{[-7,55]}\end{array}$ \\
\hline Inexplicable $(U)$ & $\begin{array}{l}15,7^{* \star * *} \\
{[6,88]}\end{array}$ & $\begin{array}{l}0,25^{* *} \\
{[2,06]}\end{array}$ & $\begin{array}{l}0,042^{*} \\
{[1,71]}\end{array}$ \\
\hline Efectos fijos: ¿ocupación? & Sí & Sí & Sí \\
\hline Efectos fijos: ¿municipio? & Sí & Sí & Sí \\
\hline Observaciones & 1531 & 1092 & 4172 \\
\hline
\end{tabular}

Estadísticas t entre paréntesis. La columna 1 utiliza MCO. Las columnas 2 y 3 muestran los efectos marginales de las regresiones Probit. ${ }^{*},{ }^{* *}, \mathrm{y}^{* * *}$ denotan niveles del 10,5 y $1 \%$ de significancia, respectivamente. 
las diferencias de género podrían potencialmente sesgar los resultados de la estimación de la ecuación (2). Heckman (1979) recomienda corregir el sesgo de selección a través de un proceso de 2 pasos. Los modelos suponen que los hombres y las mujeres tienen diferentes probabilidades de participar en mercados laborales debido a sus diferentes salarios de reserva. Estos, en cambio, se espera que sean una función del número de niños en el hogar.

A lo largo de las diversas metodologías econométricas, este estudio sigue a Grossman (1983) e incluye efectos a nivel de municipio y de ocupación. Esto se ha convertido en una práctica estándar en modelos de descomposición Blinder-Oaxaca a fin de aumentar el poder explicativo de los componentes observados $\mathrm{y}$, de ese modo, reducir el factor inexplicable que normalmente se atribuye a la discriminación. Los efectos fijos a nivel de municipio capturan la heterogeneidad no observada que puede estar presente en determinados mercados laborales dentro de algunos sectores geográficos. Por ejemplo, los municipios en zonas costeras y rurales tienen mayor dependencia agrícola y pueden experimentar una mayor ciclicidad en el empleo que los municipios serranos. El análisis utiliza 221 diferentes efectos fijos de ciudad, dándonos alrededor de 33 observaciones por municipio ${ }^{8}$. En segundo lugar, se utilizan efectos fijos para capturar características no observadas que pueden estar relacionadas con determinados tipos de trabajo. Por ejemplo, los trabajadores agrícolas pueden observar un mayor grado de carácter cíclico y mayor informalidad que los trabajadores domésticos. Los tipos de trabajo controlados son: trabajador del gobierno, trabajador privado, obrero, jefe, trabajador por cuenta propia, trabajador agrícola, jefe agrícola y trabajador doméstico. La inclusión de trabajadores del gobierno es importante, dado que Panizza y Qiang (2005) encuentran que en Latinoamérica, incluyendo Ecuador, existe una prima salarial asociada con el trabajo en el sector público. Ese estudio también encuentra que la prima suele ser mayor para las mujeres que para los hombres, pero que esta diferencia no compensa la brecha salarial de género. Además, cuando se realizó la encuesta el gobierno era el cuarto empleador más importante del Ecuador. La figura A2, en el Apéndice 1, enseña el porcentaje de trabajadores bajo cada una de las clasificaciones utilizadas en este estudio.

Finalmente, es importante señalar que, como se mencionó en la sección anterior, el número de observaciones varían significativamente a través de las regresiones cuando se emplean las diversas variables dependientes. Esto se debe a que algunos de los encuestados no sabían cuántas horas trabajan, mientras que otros no supieron contestar si tenían un contrato o no dijeron si estaban subempleados. Es posible que el tener un número variable de observaciones en las regresiones genera un sesgo. Una forma de comprobar si este es el caso es estimar la ecuación utilizando únicamente observaciones para la muestra de individuos que han respondido a todas las preguntas. Aunque esto reduce significativamente el número de observaciones, los resultados (disponibles a petición) siguen siendo ampliamente compatibles.

\footnotetext{
${ }^{8}$ Aparte de la región en donde se encuentran los municipios, es imposible verificar cuál número pertenece a qué municipio en esta base de datos.
}

\section{Resultados}

La tabla 2 muestra el primer conjunto de resultados. La columna 1 de la tabla 2 muestra que, en promedio, ser mujer disminuye los salarios por 15 centavos por hora. Esto es después de controlar por factores tales como la tenencia, edad, educación, estado civil, número de hijos, y después de incluir efectos fijos por municipio y ocupación. Para poner esto en perspectiva, basada en una jornada de 8 horas, esta diferencia constituye el $12 \%$ de la canasta mínima necesaria mensual de consumo en Ecuador, que se calcula como \$260 por mes en 2006. Del mismo modo, la columna 3 muestra que en 2006 las mujeres encuestadas en Ecuador tenían un 40\% más probabilidades de no encontrar empleo que los hombres. La columna 2, sin embargo, indica que las mujeres en la encuesta tuvieron un $7 \%$ menos probabilidades de clasificarse a sí mismas como subempleadas, en comparación con los hombres. Por último, la columna 4 revela que ser mujer en el mercado laboral de Ecuador no disminuye significativamente la probabilidad de estar empleada formalmente.

En cuanto a las demás variables, se encuentra que los pueblos indígenas tienen un $25 \%$ más de probabilidad de ser empleados y un $10 \%$ menos probabilidad de ser subempleados. Hall y Patrinos (2006) argumentan que existe una diferencia de empleo porque las personas indígenas son generalmente más pobres y, por lo tanto, tienen una opción de trabajo-ocio más restringida. Las diferencias en el subempleo, por otro lado, posiblemente reflejan niveles inferiores de logros educativos en sectores predominantemente indígenas.

Se encuentra que la tenencia aumenta significativamente los salarios por hora, por 0,19 centavos de dólar por hora por cada año adicional de empleo en una empresa. Sin embargo, el efecto de esta variable sobre el subempleo, el empleo y la formalidad es estadísticamente insignificante. Del mismo modo, la educación aumenta los salarios por hora, aunque tiene un efecto insignificante sobre las probabilidades de sentirse subempleado, estar empleado o tener un contrato. Por último, las personas que afirmaban haber estado enfermas en el último mes también ganan menos, tienen una mayor probabilidad de ser subempleadas y fueron significativamente más propensas a trabajar.

La tabla 2 indica que las mujeres están en peores condiciones que los hombres; sin embargo, esta tabla es incapaz de identificar y cuantificar las fuentes subyacentes de las diferencias de género en los resultados del mercado laboral. La tabla 3 presenta un análisis de los factores que contribuyen a la brecha de género utilizando la descomposición Blinder-Oaxaca. Los resultados en el panel A informan las predicciones de la descomposición medias por género. En la columna 1, la media de los salarios por hora es de 83 y 54 centavos de dólar por hora de hombres y mujeres, respectivamente. Esto produce una brecha salarial de 29 centavos de dólar por hora. En el panel B, la brecha salarial se divide entre las 2 partes que se discuten en la sección anterior. La primera fila del panel B muestra que 14 centavos, o aproximadamente el $50 \%$ de la brecha salarial, se pueden explicar por las diferencias en variables como la educación y la ocupación. El componente no explicado muestra que el $50 \%$ restante de la brecha salarial se puede interpretar como derivada de la discriminación por motivos de género. El panel c de la columna 1 presenta los aportes individuales 


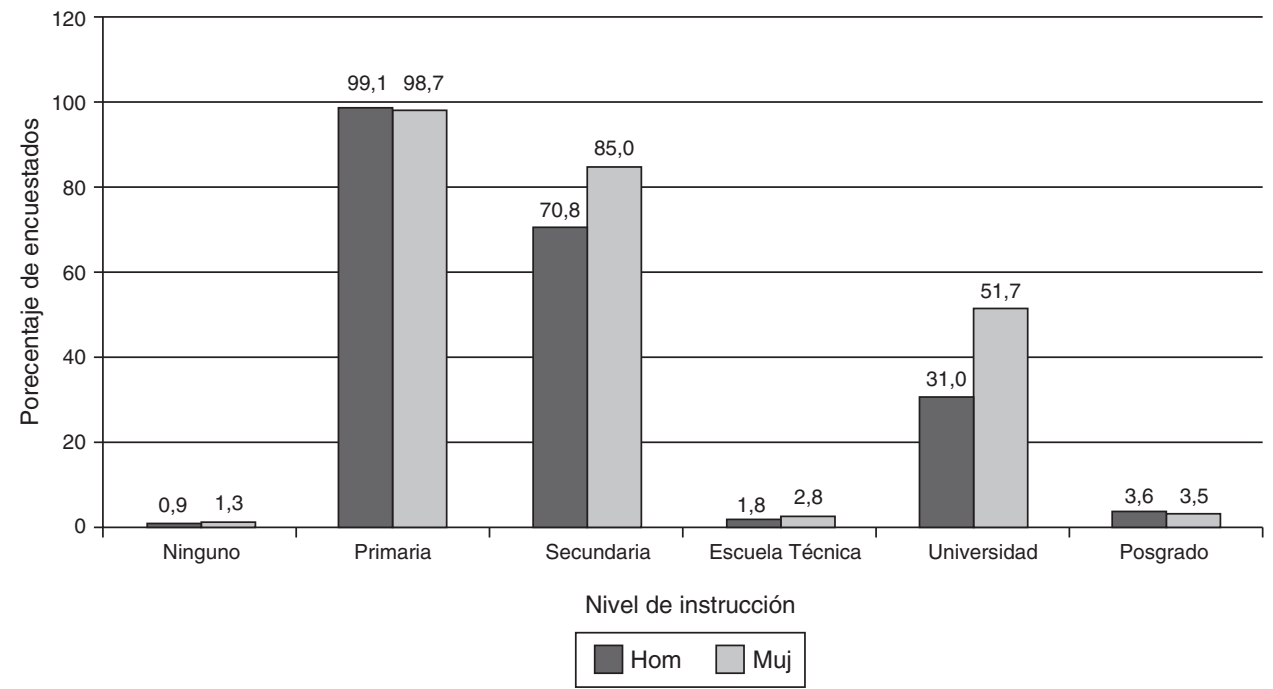

Figura 2 Nivel de instrucción entre hombres y mujeres en Ecuador, 2005-2006.

Fuente: Encuesta de Condiciones de Vida (2005-2006).

de las diferencias de género en la tenencia, edad, estado civil, educación, niños, región y ocupación. La contribución de un conjunto de variables binarias, disponibles bajo petición, muestra que las diferencias en la ocupación explican aproximadamente el $15 \%$ de las diferencias en la brecha salarial, mientras que los efectos de municipio explican aproximadamente el $30 \%$ de esta diferencia, con algunos municipios que exhiben efectos positivos, mientras que otros demuestran efectos negativos. Entre los otros indicadores, haber tenido una enfermedad en el último mes representa 0,48 centavos o $1,6 \%$ de la brecha salarial entre hombres y mujeres. Además, el 1,8\% de la brecha se explica por el número de hijos, aunque el signo del coeficiente sugiere que tener un hijo más en el hogar disminuye, en lugar de aumentar, la brecha salarial por aproximadamente 0,5 centavos de dólar por hora. Finalmente, después de los efectos fijos por municipio y ocupación, el mayor factor que explica la disparidad de género en los salarios por hora es la educación. Los diferentes niveles de educación entre las mujeres representan 0,75 centavos o un 2,6\% de la brecha salarial. Este porcentaje pequeño sugiere que no es probable que las políticas que aumentan el nivel de instrucción de las mujeres resulten en una reducción significativa de la brecha salarial de género en el Ecuador ${ }^{9}$. Esto no es sorprendente, dado que los hombres y las mujeres tienen los mismos niveles de educación primaria y que las mujeres superan a los hombres en cuanto a la educación secundaria y universitaria (véase fig. 2).

Sin embargo, es importante tener en cuenta que las cifras de logro educativo ignoran las diferencias en el tratamiento de niños y niñas en el aula y la transmisión de estereotipos sexistas a través de programas de estudio y los textos escolares (UNESCO, 2002). Esto tiene 2 implicaciones serias para este estudio. En primer lugar, esto sugiere que las políticas educativas en Ecuador deben tratar de deshacer

\footnotetext{
9 García-Aracil y Winter (2006) llegan a una conclusión similar en su estudio.
}

algunos de estos sesgos existentes, por ejemplo, el ajuste de los planes de estudios para especificar las aportaciones de las mujeres en el desarrollo cultural y social (Subirats, 2006). En segundo lugar, esto sugiere que el análisis econométrico puede atribuir los resultados del mercado laboral como resultado de las diferencias educativas a discriminación en el mercado laboral, en lugar de prejuicios culturales en el sector de la educación, lo que genera un sesgo a la baja en las estimaciones de los coeficientes adjuntos a la educación y un sesgo al alza en el efecto de la discriminación.

En cuanto a las variables dependientes restantes, la columna 2 de la tabla 3 indica que los hombres y las mujeres tienen la misma probabilidad de ser autoclasificados como subempleados. La columna 3 indica que las mujeres son significativamente menos probables de encontrar empleo que los hombres (la diferencia entre las tasas de empleo de hombres y mujeres es del $30 \%$ ). Por otra parte, la descomposición en el panel $B$ sugiere que esta diferencia se debe a la discriminación, ya que ninguno de los factores explicados captura de forma significativa esta diferencia. Este punto se trata en más detalle más adelante; sin embargo, es importante señalar que la magnitud de este efecto puede ser debida a una variable de productividad omitida que se correlaciona positivamente con el género.

Por último, la columna 4 de la tabla 3 indica que las mujeres son más propensas a tener un contrato que los hombres. Curiosamente, los resultados muestran un efecto explicado negativo y un componente sin explicación positivo. En otras palabras, el componente explicado muestra que las diferencias en las características de algunas mujeres, como la ocupación, aumentan significativamente la probabilidad de estar empleadas formalmente por aproximadamente un $10 \%$. Sin embargo, el componente no explicado indica que hay un $6 \%$ más de probabilidades de que las mujeres trabajen de manera informal que los hombres, debido a factores que pueden ser interpretados como discriminación de género.

Una razón por la que nos encontramos con que las mujeres son más propensas a tener un contrato que los hombres 
puede que se deba al hecho de que los trabajadores en sectores dominados por las mujeres, tal como la limpieza y la horticultura, son más propensos a tener un contrato que los trabajadores que utilizan a los hombres como factor intensivo, tal como la agricultura y la construcción ${ }^{10}$. De hecho, entrevistas con un órgano de representación de las empresas exportadoras de flores, donde predominan las mujeres, mostró que la adhesión a las normas internacionales del trabajo es importante para muchos de los importadores europeos y americanos ${ }^{11}$. Aparte de la ubicación y la ocupación, el mayor factor que explica esta discriminación positiva a favor de las mujeres es la educación. Las diferencias en la educación entre los hombres y las mujeres explican el $42 \%$ de la diferencia en formalidad de empleo. Sin embargo, la columna 4 también indica que las diferencias de grupo en las características de familia, tal como estar casado, contribuyen positivamente hacia la brecha hombre/mujer de la formalidad de empleo: las mujeres casadas tienen un $0,4 \%$ menos probabilidades de ser empleadas formalmente que los hombres casados. Esto sugiere que, tal vez, las responsabilidades del hogar están obligando a las mujeres a optar hacia menos oportunidades de empleo formal. Este punto se trata en más detalle a continuación.

Los resultados de la estimación de la ecuación (2), tomando en cuenta la corrección de sesgo de Heckman, están en la tabla 4. Para facilitar la exposición, los resultados no incluyen la descomposición del efecto explicado (panel $C$ de la tabla 3). Téngase en cuenta, sin embargo, que los resultados de esta descomposición son consistentes con los de la tabla anterior. La tabla demuestra que incluso después de tener en cuenta las diferencias en el conjunto de elección disponibles para hombres y mujeres, existen diferencias significativas en las 4 variables dependientes. En cuanto a los salarios y la formalidad, los resultados de la tabla 4 (columnas 1 y 4) son consistentes con los de la tabla 3. Además, la tabla 4 también encuentra evidencia estadísticamente significativa de que los hombres y las mujeres difieren en términos de resultados de subempleo y de empleo. La columna 2 revela que los hombres son un $23 \%$ más propensos a autoidentificarse como subempleados en comparación a las mujeres. Por otra parte, después de controlar por la educación, la ocupación y otros componentes de $Q$, la columna revela que esta autoidentificación se debe a la discriminación de género. Del mismo modo, la columna 3 muestra que las mujeres son el $37 \%$ menos propensas a ser empleadas, lo cual también se debe a factores inexplicables.

La discriminación, en este caso, se puede interpretar como que las mujeres tienen diferentes percepciones sobre sí mismas en el mercado laboral que los hombres. La tabla 4 muestra evidencia de que se puede interpretar como que las mujeres buscan menos empleo, menos empleo formal y menos horas de trabajo que los hombres. Maloney (2004) sostiene que las mujeres buscan estos resultados con el fin de lograr una mayor flexibilidad en las horas de

\footnotetext{
10 La clasificación de ocupación no entra en esta profundidad de detalle, por lo que solo se puede especular que este es el caso.

11 La evidencia de la importancia de adherirse a las normas y códigos para los exportadores internacionales del trabajo se discute en detalle por Belwal y Chala (2008) y Melese y Helmsing (2010) para el caso de Etiopía.
}

trabajo para que puedan cumplir con sus compromisos en el hogar. Esta decisión podría deberse a la presión sociocultural sobre las mujeres, en lugar de decir sobre las decisiones que pueden maximizar los ingresos del hogar o de la utilidad. Abordar los factores socioculturales que pueden llevar a esta forma de discriminación es probablemente el mayor desafío de Ecuador.

Es importante tener en cuenta la posibilidad de que, al igual que los hombres y las mujeres potencialmente pueden diferirse, los resultados de la tabla 4 también pueden sufrir de un sesgo generado por el hecho de que los salarios, el subempleo y la formalidad se centran exclusivamente en las personas que han decidido entrar en el mercado laboral. Es decir, los resultados del mercado laboral se observaron solamente para las personas que están empleadas, y esto puede ser un grupo selectivo. Para hacer frente a este sesgo potencial, la tabla 5 emplea una descomposición adicional Heckman, que en este caso sigue un proceso de 3 pasos. El primer paso modela la decisión de una persona de trabajar en función de su edad, la edad al cuadrado y el nivel de educación de su madre -este último se ha encontrado como uno de los determinantes de resultados laborales más influyentes (Haveman y Wolfe, 1995). La segunda y tercera parte emplean una corrección estándar de Heckman, al igual que en la tabla 4. En general, los resultados de la tabla 5 son consistentes con los que se encuentran en la tabla $4^{12}$.

\section{Conclusión}

Estudios anteriores sobre la discriminación hacia las mujeres en los mercados laborales se concentran únicamente en brechas salariales. Este estudio hace una contribución especial a esta literatura al también estudiar si existen brechas de género en términos de empleo, subempleo y formalidad de empleo. Utilizando datos de un estudio exhaustivo de hogares en los años 2005-2006, se encuentra evidencia de que la discriminación en contra de la mujer en los mercados laborales vas más allá de los sueldos.

Los resultados del análisis usando MCO muestran que, en promedio, ser mujer disminuye los salarios por casi el $20 \%$, mientras que las descomposiciones utilizando los métodos de Blinder-Oaxaca, Heckman y Heckman doble demuestran que el $50 \%$ de la brecha salarial se puede interpretar como discriminación por motivos de género. Se argumenta que es poco probable que un aumento en el acceso a la educación para las niñas aborde estos problemas.

Entonces, otra forma posible de disminuir esta brecha es la introducción de una legislación efectiva de igualdad de remuneración. Sin embargo, los resultados en este trabajo también revelan evidencia de discriminación en contra de la mujer en términos de ofrecer (o buscar) contratos formales. Aunque los resultados del análisis utilizando MCO demuestra que ser mujer no disminuye la probabilidad de estar empleada formalmente, los componentes no explicados en Blinder-Oaxaca, Heckman y doble Heckman indican que las

\footnotetext{
12 Tenga en cuenta que en la estimación de esta ecuación doble Heckman, edad, edad al cuadrado, número de hijos y estudios de la madre se eliminan de la ecuación en la última etapa con el fin de garantizar que las ecuaciones se identifican (Puhani, 2000).
} 
mujeres tienen un 6\% más de probabilidad de trabajar de manera informal que los hombres debido a factores que pueden ser interpretados como discriminación de género. Por lo tanto, la legislación sobre igualdad de remuneración podría aumentar esta tendencia, ya que las empresas tratan de evitar ofrecer contratos formales con el fin de pagar salarios más bajos. Para evitar este problema, el gobierno ecuatoriano podría introducir incentivos fiscales para las empresas que muestran que están activamente corrigiendo los desequilibrios de género mediante la contratación de más mujeres y garantizando la igualdad de remuneración.

Por otra parte, una interpretación de los resultados de este trabajo es que las mujeres eligen no estar empleadas o trabajar menos horas. Los resultados del análisis con MCO demuestran que las mujeres tenían un $40 \%$ más probabilidades de no encontrar empleo que los hombres. La descomposición Blinder-Oaxaca, Heckman y Heckman doble sugieren que esta diferencia se debe enteramente a factores no observados. Similarmente, las mujeres tienen un 7\% menos probabilidades de clasificarse a sí mismas como subempleadas, mientras que la descomposición Heckman y Heckman doble demuestran que los hombres son un $23 \%$ más propensos a autoidentificarse como subempleados, y que esta diferencia se debe a la discriminación de género. Es decir, que las mujeres buscan menos empleo formal que los hombres. Algún componente, entonces, de la discriminación puede deberse a que las mujeres valoran sus contribuciones en el mercado laboral menos que los hombres. Se argumenta que esto puede deberse a que las mujeres tienen relativamente más responsabilidades domésticas. Esto significa que las diferencias de género en Ecuador posiblemente se deben a factores socioculturales que hacen el empleo formal de tiempo completo menos atractivo. Algunos analistas sugieren que la autoselección en esta forma de empleo se debe a que las mujeres pueden equilibrar más fácilmente sus roles de producción (mercado) y reproductivos (atención domiciliaria). El problema, sin embargo, es que, al hacerlo, los países en desarrollo, como el Ecuador, están renunciando a la utilización eficiente de la capacidad productiva de la mitad de su población. Lo ideal es que los hombres y las mujeres puedan participar de manera más equitativa en los roles domésticos, incluyendo la crianza de los niños, ayudando a que las mujeres estén más dispuestas y capaces de participar en el empleo formal a tiempo completo. Por lo tanto, las políticas dirigidas a promover la igualdad en el hogar mediante la enseñanza de los beneficios del empleo formal, por ejemplo, podrían ser beneficiosas.

Por otra parte, las reformas como el Mandato $\mathrm{N} .{ }^{\circ} 8$, cuyo objetivo es formalizar el mercado laboral, pueden llegar a ser fructíferas. Es por esto interesante volver a analizar este tema utilizando una base de datos similar para entender mejor si algunas de las políticas encabezadas por el gobierno actual han dado resultados. Dado que los mercados laborales cambian muy lentamente, como es argumentado en este estudio, sería muy importante estudiar las brechas de género en mercados laborales cada 5 a 10 años. Sería, por esto, también muy útil introducir un estudio longitudinal en el mercado laboral ecuatoriano. Un panel de observaciones longitudinales también nos pudiera ayudar a mejor controlar factores no explicables de la descomposición dándonos resultados más precisos.

\section{Apéndice 1.}

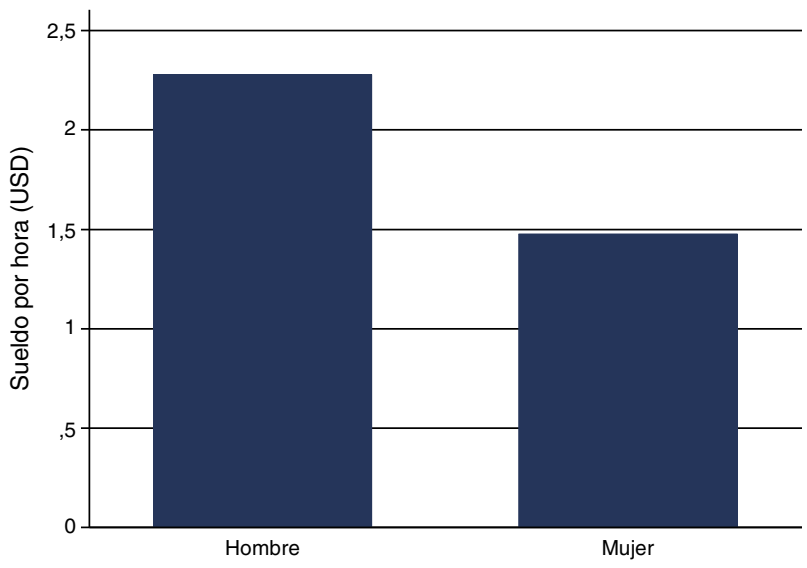

Figura A1 Sueldos por género en Ecuador, 2012.

Fuente: INEC (2012).

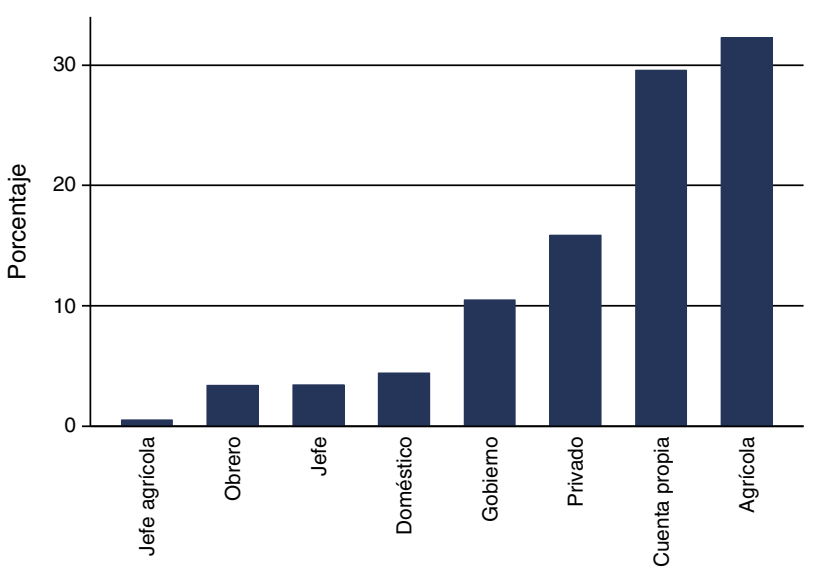

Figura A2 Clasificación de trabajadores según tipos de trabajo.

Fuente: Encuesta de Condiciones de Vida (2005-2006).

\section{Bibliografía}

Abramo, L., 2004. Inserción laboral de las mujeres en América Latina: una fuerza de trabajo secundaria. Revista Estudios Feministas. 12, 224-235.

Altonji, J.G., Blank, R., 1999. Race and gender in the labor market. Handbook of Labor Economics. 3, 3143-3259.

Altonji, J.G., Dunn, T.A., 1996. The effects of family characteristics on the return to education. The Review of Economics and Statistics. 78, 692-704.

Arizpe, L., 1977. Women in the informal labor sector: The case of Mexico City. Signs 3, 25-37.

Atal J., Nopo H. Winder N., 2009. New Century, Old Disparities: Gender and Ethnic Wage Gaps in Latin America. IDB Working Paper. No. 25.

Basabe-Serrano, S., 2013. Explicando la corrupción judicial en las cortes intermedias e inferiores de Chile, Perú y Ecuador. Perfiles Latinoamericanos 21, 79-108. 
Belwal, R., Chala, M., 2008. Catalysts and barriers to cut flower export: A case study of Ethiopian floriculture industry. International Journal of Emerging Markets. 3, 216-235.

Blau, F.D., Brummund, P., Liu, A.Y.H., 2013. Trends in occupational segregation by gender 1970-2009: Adjusting for the impact of changes in the occupational coding system. Demography 50, 471-492.

Blinder, A., 1973. Wage discrimination: Reduced form and structural estimates. Journal of Human Resources. 8, 436-455.

Calla, R., Milosavljevi, V., 2007. La Mujer Indígena en Bolivia, Brasil, Ecuador, Guatemala y Panama: Un Panorama de Base a Partir de la Ronda de Censos de 2000. Serie Mujer y Desarrollo. Consejo Económico para América Latina y el Caribe, Santiago.

Cano, D., 2010. Regresión laboral en el Ecuador y sus consecuencias: Gobierno de Rafael Correa, en: ¿Estado constitucional de derechos? Informe sobre derechos humanos Ecuador 2009. Universidad Andina Simón Bolívar. Sede Ecuador. Programa Andino de Derechos Humanos, PADH; Abya Yala, Quito, pp. 291-314.

Chun, H., Lee, I., 2001. Why do married men earn more: Productivity or marriage selection? Economic Inquiry. 39, 307-319.

Dávalos X., 2012. Credibilidad de la Política Fiscal en el Ecuador, XXIV Congreso Sobre Política Fiscal, Santiago, Ene 2012: CEPAL.

de Barros, R.P., 2009. Measuring Inequality of Opportunities in Latin America and the Caribbean. World Bank Publications, Washington.

Deere, C.D., León, M., 2003. The gender asset gap: Land in Latin America. World Development 31, 925-947.

Fairlie, R.W., 1999. The absence of the African-American owned business: An analysis of the dynamics of self-employment. Journal of Labor Economics. 17, 80-108.

Fairlie, R.W., 2005. An extension of the Blinder-Oaxaca decomposition technique to logit and probit models. Journal of Economic and Social Measurement. 30, 305-316.

García-Aracil, A., Winter, C., 2006. Gender and ethnicity differentials in school attainment and labor market earnings in Ecuador. World Development 34, 289-307.

Grossman, J.B., 1983. The impact of the minimum wage on other wages. Journal of Human Resources 18, 359-378.

Hall, G., Patrinos, H.A. (Eds.), 2006. Indigenous Peoples, Poverty and Human Development in Latin America. Palgrave Macmillan, New York.

Haveman, R., Wolfe, B., 1995. The determinants of children's attainments: A review of methods and findings. Journal of Economic Literature, 1829-1878.

Heckman, J., 1979. Sample selection bias as a specification error. Econometrica. 47, 153-163.

Heckman J., Pagés C., 2003. Law and Employment: Lessons from Latin American and the Caribbean, NBER Working Paper 10129.

Henley, A., Arabsheibani, G.R., Carneiro, F.G., 2009. On defining and measuring the informal sector: Evidence from Brazil. World Development 37, 992-1003.

Instituto Nacional de Estadísticas y Censos (INEC), 2006. Encuesta de condiciones de vida, Quinta Ronda ECV. INEC, Quito.

Instituto Nacional de Estadísticas y Censos (INEC), 2012. Encuesta Nacional de Trabajo Infantil. INEC, Quito.

Jann, B., 2008. The Blinder-Oaxaca decomposition for linear regression models. The Stata Journal. 8, 453-479.

Kim, M.K., Polachek, S.W., 1994. Panel estimates of the gender earnings gap. Journal of Econometrics 61, 23-42.

Larrea, C., Montenegro Torres, F., 2006. Ecuador. En: Hall, G., Patrinos, H. (Eds.), Indigenous Peoples, Poverty and Human Development in Latin America. Palgrave Macmillan, Londres.

León, M., 2012. Políticas, programas y proyectos de inserción laboral para la juventud del Ecuador. Edición FLACSO and Universidad Politécnica Salesiana, Quito.

Lewis, W.A., 2013. Theory of Economic Growth, Vol. 7. Routledge, Oxford.
Lustig, N., Lopez-Calva, L.F., Ortiz-Juarez, E., 2013. Declining inequality in Latin America in the 2000s: The cases of Argentina, Brazil, and Mexico. World Development 44, 129-141.

Madrid, R.L., 2010. The origins of the two lefts in Latin America. Political Science Quarterly. 125, 587-609.

Maclsaac, D., Rama, M., 1997. Determinants of hourly earnings in Ecuador: The role of labor market regulations. Journal of Labor Economics. 15, S136-S165.

Maloney, W.F., 2004. Informality revisited. World Development. 32, 1159-1178.

Marcouiller, D., Ruiz de Castilla, V., Woodruff, C., 1997. Formal measures of the informal-sector wage gap in Mexico, El Salvador, and Peru. Economic Development and Cultural Change. 45, 367-392.

Neuman, S., Oaxaca, R.L., 2004. Wage decompositions with selectivity-corrected wage equations: A methodological note. The Journal of Economic Inequality. 2, 3-10.

Oaxaca, R.L., 1973. Male-female wage differentials in urban labor markets. International Economic Review. 14, 693-709.

Oaxaca, R.L., Ransom, M.R., 1994. On discrimination and the decomposition of wage differentials. Journal of Econometrics. 61, 5-21.

OIT, 2011. Perfiles del empleo y trabajo decente en América Latina y el Caribe. En: Jóvenes Ecuatorianos en Cifras. OIT, Ginebra.

Ortiz, I., Cummins, M., 2011. Global Inequality: Beyond the Bottom Billiom. A Rapid Review of Income Distribution in 141 Countries. UNICEF Policy and Practice, New York.

Ospina Peralta, P., 2011. Ecuador: la participación ciudadana en el proyecto de Estado de Rafael Correa. Observatorio Latinoamericano. 7, 124-145.

Pagés, C., Pierre, G., Scarpetta, S., 2009. Job creation in Latin America and the Caribbean: recent trends and policy challenges. Palgrave Macmillan/Worldbank, Washington, D.C.

Panizza, U., Qiang, C.Z.W., 2005. Public-private wage differential and gender gap in Latin America: Spoiled bureaucrats and exploited women? The Journal of Socio-Economics 34, 810-833.

Papadópulos, J., Radakovich, R., 2006. Educación superior y género en América Latina y el Caribe. En: Salazar, J.M. (Ed.), Educación superior y género: Tendencias observadas. IESALC, Caracas.

Pautassi L.C., Faur E., Gherardi N., 2005. Legislación laboral y género en América Latina. Avances y omisiones. Documento presentado en la reunión de expertos «Family-oriented policies, social protection and inclusion», Santiago, Chile, Economic Commission for Latin America and the Caribbean (ECLAC), 28-29.

Posso Serrano A., Posso A., 2009. La Función Social de la Propiedad Privada y los Incentivos de Agentes Económicos en el Socialismo del Siglo XXI. Contribuciones a la Economía. Mayo.

Puhani, P., 2000. The Heckman correction for sample selection and its critique. Journal of Economic Surveys 14, 53-68.

Subirats, M., 2006. La educación de las mujeres: de la marginalidad a la coeducación. Propuestas para una metodología de cambio educativo. En: Rodríguez, C. (Ed.), Género y currículo. Aportaciones del género al estudio y práctica del currículo. Akal, Madrid, pp. 229-255.

Salazar Maldonado D.I., 2009. El mercado laboral ecuatoriano: un análisis del impacto de las políticas públicas en el marco de las regulaciones del mandato N. ${ }^{\circ}$ 8-2008. Facultad de Economía. PUCE. Quito. 158.

Śniadecka-Kotarska M., Gil Canova P.P., 2006. Ser mujer en Ecuador (Vol. 31). Universidad de Varsovia, Centro de Estudios Latinoamericanos CESLA.

Tenjo J., Ribero R., Bernat L., 2005. Evolución de las diferencias salariales por sexo en seis países de América Latina: un intento de interpretación. Documento CEDE, No. 18. Facultad de EConomía, Universidad de los Andes. 
UNCTAD, 2012. Trade and Development Report: Evolution of Income Inequality- Different Time Perspectives and Dimensions. UNCTAD, New York and Geneva.

UNESCO, 2002. Gender Equality in Basic Education in Latin America and the Caribbean (State of the Art), Santiago: UNESCO Regional Office of Education for Latin America and the Caribbean.

USAID, Ministerio de Trabajo y Empleo (MTE), 2008. Discrimination in the Ecuadorian Labor Market. Quito: Ministerio de Trabajo y Empleo.
Weichselbaumer, D., Winter-Ebmer, R., 2005. A meta-analysis of the international gender wage gap. Journal of Economic Surveys. 19, 479-511.

Weller, J., 2011. Panorama de las condiciones de trabajo en América Latina. Nueva Sociedad 232, 32-49.

Weller J., 2012. Crecimiento, empleo y distribución de ingresos en América Latina, CEPAL - Serie Macroeconomía del desarrollo. N. 122.

Yun, M., 2004. Decomposing differences in the first momento. Economics Letters. 82, 275-280. 